\title{
Communication roles that support collaboration during the design process
}

Diane H Sonnenwald*, Risø National Laboratory, PO Box 49, Roskilde, Denmark

It is widely acknowledged that design (and development) teams increasingly include participants from different domains who must explore and integrate their specialized knowledge in order to create innovative and competitive artefacts and reduce design and development costs. Thus communication, including integration of specialized knowledge and negotiation of differences among domain specialists, has emerged as a fundamental component of the design process. This paper presents 13 communication roles that emerged during four multidisciplinary design situations in the USA and Europe. These roles supported knowledge exploration and integration, collaboration, and task and project completion by filtering and providing information and negotiating differences across organizational, task, discipline and personal boundaries. Implications for design methods, tools and education are discussed.

Keywords: collaborative design, design process, communication, teamwork

I n response to mature markets, increasing world-wide competition, fast technological development and increasing liability regulation, many companies strive to economically create innovative systems or artefacts with high volume distribution. The creation of innovative artefacts often requires the exploration and integration of dynamic and diverse knowledge from multiple domains, disciplines and contexts among specialists. For example, many engineering firms now use concurrent engineering design methods that emphasize the integration of engineering, manufacturing, marketing and distribution, maintenance and repair, disposal and recycling, and application (end-user) knowledge. Similarly information systems projects include specialists in database management, human-computer interaction, software engineering, documentation and

* Currently at School of Information and Library Science, 100 Manning Hall, CB 3360, The University of North Carolina at Chapel Hill, Chapel Hill, NC 27599-3360, USA

0142-694X/96 \$15.00 Design Studies 17 (1996) 277-301 0142-694X(96)00002-6 (C) 1996 Elsevier Science Lid Printed in Great Britain All rights reserved 
Figure I Knowledge or ploration dormg the design process
1 Rasmussen, J, Peftersen, A and Goodstaln, L Cognitive systems engineering Wiley, New York (1994)

2 Bucciarelii, L An ethnographic perspective on engineer. ing design' Design Studies Vol 9 (1988) 159-168

3 Krippendort, $K$ 'On the essential contexts of antifacts or on the propostion that "Design is making sense (of things)" $D$ e. sign issues vol 5 No 2 (1989) 9-39

4 Beer, $S$ Designing freedom Wiley. New York (1974)

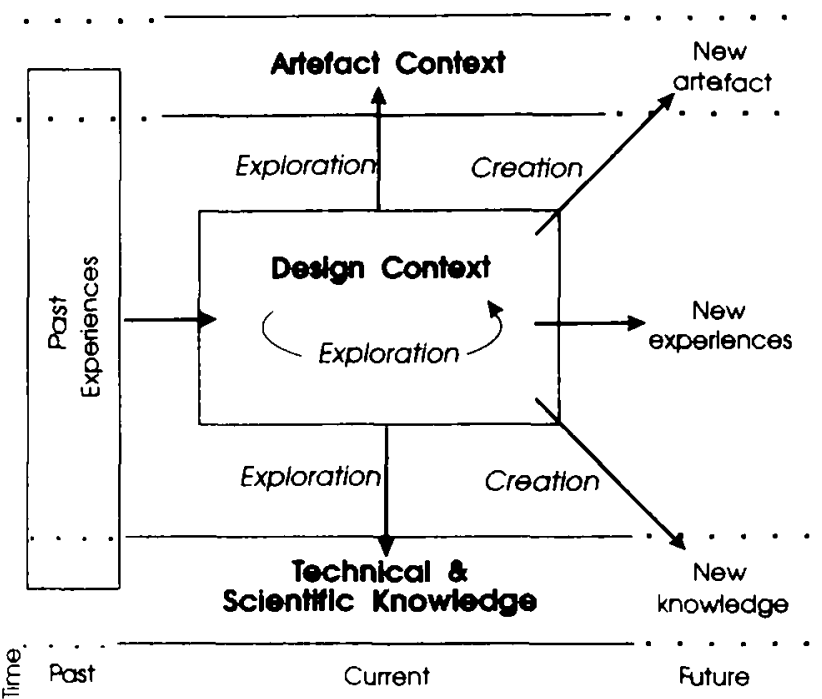

training. telecommunications and end-user domains. During the design process (see Note 1) these specialists build on their past experiences with artefact contexts, design contexts, or situations, and technical and scientific knowledge (see Figure 1). They explore and integrate knowledge about the current (and evolving) artefact context. design context, and technical and scientific knowledge. The expected outcomes include the creation of an artefact to be useful in the future artefact context, perhaps the creation of new technical and scientific knowledge, and knowledge about the design process to be applied to new design experiences.

Exploration of the artefact context is required to understand how the artefact should support patterns of work tasks, organization structures, social groups and individual preferences ${ }^{1.2}$ as well as the artefact's potential impact on the environment, culture, individual perceptions of reality, and other artefacts and system $s^{3.4}$. Each artefact context can, of course, consist of multiple contexts. For example, a library information system context may consist of the library policy, management, material acquisitions, reference, and patron, or client, services contexts. A challenge in design is to explore and integrate knowledge about these contexts so the system, or artefact, will support activities and values in each of these domains. When artefact contexts are not explored. design decisions may have a negative impact on users. 
5 Sonnenwald, D H Communication in design' PhD thesis, Rutgers, The State University of Now Jorsey (1993)

6 Llewroum, $L$ and Finn, $T$ 'Identifying the common dimensions of communication: The communication systems madel' in B D Ruben and $L A$ Levroum (eds) Modiation, information, and communication: Information and behevior Vol. 3, Transaction Publishers, Now Brunswick, NJ (1990) p 49

7 coftman, E Encounters The Bobbs-Merrill Co., Inc., New York (1961) p 85
Furthermore, the design context itself must be explored during the design process. Increasingly design teams include participants from different disciplines, organizations and cultures because the creation of innovative artefacts may require specialists from a variety of disciplines and contexts not all of whom may work in one organization, company or country. These participants come to the design situation with pre-existing patterns of work activities, specialized work languages, and different expectations and perceptions of quality and success, and different organizational constraints and priorities. Design participants need to explore and integrate these differences. When the design context is not explored, project team members may make design decisions that have a negative impact on other members' work and on the artefact as a whole.

Similarily, exploration of technical and scientific knowledge may be required for a variety of reasons including finding solutions to design problems, data to support intuitive solutions, and methods to help interpret the artefact context. To create innovative artefacts, design participants must increasingly explore technical and scientific information from a variety of disciplines.

However, knowledge exploration can be difficult for design participants. Participants' unique past experiences, specialized work language, and differences in work patterns, perceptions of quality and success, organizational priorities, and technical constraints may cause them to challenge or contest one another's contribution. This phenomenon, characterized as 'contested collaboration', can lead to conflict and has a negative impact on the quality of the design process and design outcomes. If we understand the process of successful knowledge exploration and collaboration in design, we can perhaps better prepare our students who may one day participate in multidisciplinary design teams, better understand how tools could support knowledge exploration and collaboration, and create predictive models that facilitate knowledge exploration and collaboration during the design process.

\section{Research approach}

An important aspect of knowledge exploration and collaboration is communication. The definition of communication used in this research is human behaviour that facilitates the sharing of meaning and which takes place in a particular social context ${ }^{.6}$ and a role is defined as "the activity the incumbent would engage in were he to act solely in terms of normative demands upon someone in his position" ${ }^{7}$. An individual may perform one or more roles and may change roles. Role performance, or role enactment, is the actual conduct of an individual while assuming a role. Role 
8 Fisher, $B$ and Ellis, D Small group decision making: Communication and the group pro. cess, McGraw Hill. New York (1990) p 293

9 Allon, $T J$, Lee, $D M$ and Tushmen, M L 'R8D perform. ance as a function of internal communication, project management, and the nature of work IEEE Transactions on Engineer. ing Management Vol EM-27 (1980) 2-12

10 Tushman, M L Special boundary roles in the innovation process Administrative Scrence Quarterly, Vol 22 (1977) 587-605 11 Tushman, $M L$ and Katz. R External communication and project pertormance: An investigation into the role of gatekeepers Management Science Vol 26 No 11 (1980) 1071-1085

12 Heuptman, 0 'Influence of task type on the relationship between communication and per. formance: The case of sotware development R8O Management Vol 16 (1986) 127-139 performance occurs primarily through interaction with others. Others have expectations with respect to an individual's role performance and these expectations help to shape an individual's behaviour. The individual's performance, in particular their communication behaviour, is the elementary unit of analysis in role theory. By observing communication behaviour among design participants we can begin to understand the roles that emerge during the design process that enable participants to mutually explore and integrate knowledge.

A type of role that appears to be of particular importance to knowledge exploration and collaboration in design is the boundary spanning role. A boundary spanning role is defined as communication and information processing behaviour between two or more networks" or groups. Boundary spanning roles have been studied in other organizational contexts" 11 and results from these studies positively relate high boundary spanning activity to high project performance in a variety of organizational settings. Boundaries are typically presented as formal project, department (or laboratory), and company (or corporate) boundaries. Boundary spanning roles which have been identified include: the internal star (individuals whose interaction with their project or department or organization members occurred more frequently than average), external star (individuals who had a high frequency of communication external to their project), and gatekeeper (individuals who had a high frequency of interaction both outside and inside their projects). However, differences duc to task type, task process and organizational context appear to influence role characteristics and project and individual performance. For example. Hauptman ${ }^{12}$ proposed that boundary spanning activity in software development is not similar to boundary spanning activity in development or research projects in $R \& D$ organizations based on the results of a sociometric study of software development projects.

Building on these results I conducted studies to identify boundary spanning roles and strategies that emerge during the design process.

\subsection{Related research}

Because not much is known about communication during the design process, recent research. in particular research on communication during the software and enginecring design process (the primary focus of this paper) has utilized an empirical and grounded theory approach, utilizing data from actual design participants and situations to develop models and theories. As illustrated in Table 1, this research can be classified into three categories based on their research approach. 


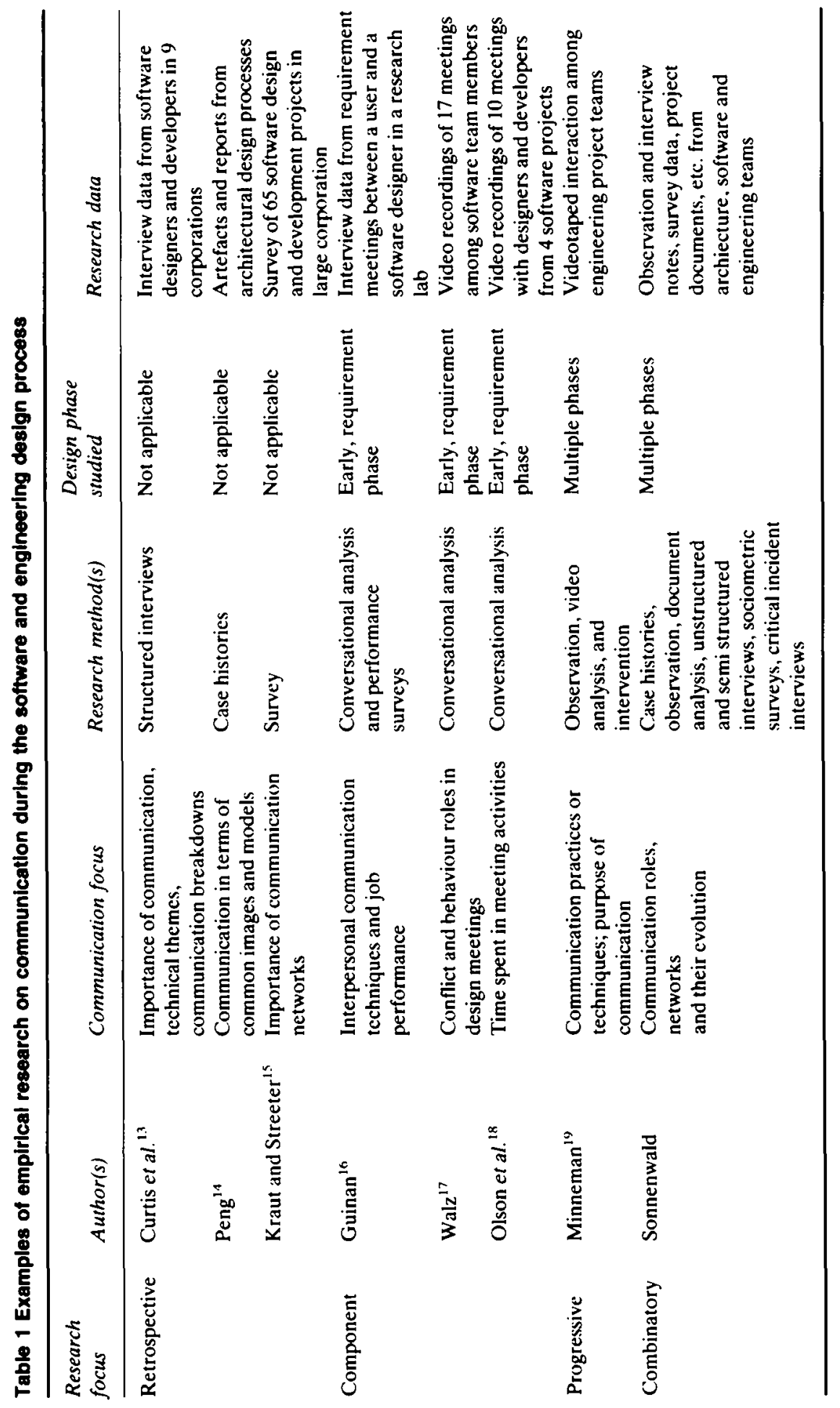


13 Curtis, $B$, Krasenor, $H$ and lecoe, N 'A field study of the software design process for large systems ' Communications of the ACM Vol 31 (1988) 1268-1287 14 Peng, $C$ Exploring communication in collaborative design: $\infty$-operative architectural modelling' Design Studies Vol 15 No 1 (1994) 19-44

15 Kraut, R E and Streoter, L A Coordination in software de vetopment' Communications of the ACM Vol 38 No 3 (1995) $69-81$

16 Gulnen, $P$ Spectalistgeneralist communication competence: A field experiment investigating the communication bohavior of information systems devetopers' PhD thesis, Indiana University, Bloomington. IN (1986)
The first category is termed 'retrospective': research in this category examines retrospective and historical accounts of the design process as provided in design participants retrospective descriptions of communication activities and histories of the design process. In this way, these studies analyse communication using participants' reconstruction of their realities. Examples of studies that have taken this approach include Curtis et $a^{13}{ }^{13}$, Peng ${ }^{14}$, and Kraut and Streeter ${ }^{15}$.

Curtis $e$ al. ${ }^{1.3}$ conducted one-hour-long structured interviews with 97 designers and developers from nine corporations involved in 19 projects in stages ranging from early planning through to maintenance. They reported the three most salient problems in design for the designers and developers were: the thin spread of application domain knowledge, fluctuating and conflicting requirements, and communication and coordination breakdowns. Factors influencing communication breakdowns included communication skills of individuals, existing incentive systems, different representational formats, rapid change. local jargon, breakdown of information capture (i.e., overwhelming amounts of information), and cultural mores and norms for individual behaviour.

Peng ${ }^{\text {th }}$ analysed three cases of architectural modelling and proposed that communication among design team members may be characterized in terms of interrelationships between common images shared by the group and domain design expressions known by individuals. Peng suggested that design team members creatc artefacts by representing, mapping, constructing and querying these images and expressions.

Another recent, retrospective study surveyed 65 design situations within a corporation to discover how designers and developers communicated and co-ordinated software development. Kraut and Streeter ${ }^{15}$ found that extensive interpersonal networks appeared to improve design outcomes. especially in projects with a high degree of uncertainty. Design situations with denser cross-boundary networks were better informed and coordinated, and the use of formal procedures did not seem to improve intergroup co-ordination.

The second category of studies summarized in Table 1 is called component'. These studies focus on components, or phases, of the design process, and thus view a 'slice' of communication behaviour during the design process. For example, Guinan ${ }^{16}$ investigated communication during requirement interviews between software designers and users (in laboratory settings) and its relationship to designer performance. She proposed that several interpersonal communication techniques, such as 
17 Warz, D A longitudinal study of the group design process' $\mathrm{PhD}$ thesis. The University of Texas at Austin (1988)

18 Oteon, G, Oleon, J, Carter, $M$ and Storrecten, M 'Small group design meotings: an analy. sis of collaboration' HumanComputer interaction Vol 7 (1992) 347-374

19 Minneman, $s$ 'The social construction of a technical reality: ompinical studies of group ongineering design practico" PhD thesis. Stanford University (1991) metacommunication, reframing and backtracking, are positively related to designer performance.

Walz ${ }^{17}$ also focused on the early phases of the design process but studied intragroup communication among designers. She did a conversation analysis of 17 meetings attended primarily by designers over four months during the early phase (software requirement specification stage) of an object-oriented database management system. Walz observed conflict and uncertainty in these meetings and suggested that conflict among designers was high as they attempted to determine design requirements, decreased when they communicated the determined requirements to users, and increased as they discussed how to fulfil the requirements.

Olson et al. ${ }^{1 \mathrm{R}}$ expanded Walz' study by analysing design meetings from four software teams. Similarly to Walz, they carried out a conversational analysis of videotaped meetings but focused on the time spent in meeting activities. They found similar patterns across design teams. In meetings, teams spent only $40 \%$ of the time in direct discussions of design, with swift transitions between alternative ideas and their evaluation. $30 \%$ of meeting time was spent taking stock of their progress, and co-ordination activities consumed approximately $20 \%$ of the time. Clarification of ideas across these activities took one-third of the time, indicating that participants spent a large amount of time sharing and explaining expertise.

The third category of empirical research that focuses on communication is termed 'progressive', in that it investigates the design process as it unfolds, or evolves, over time. For example, research by Minneman ${ }^{19}$ investigated communication across multiple design phases. In particular, Minneman observed engineering teams, analysed video recordings of team interaction, and intervened in the design process by sharing his insights into their communication with design team members. Minneman suggests that the practices of negotiating understandings, conserving ambiguity, tailoring engineering communication for recipients, and manipulating mundane representations are important strategies used by design participants.

The research reported here incorporates aspects of the retrospective, component and progressive research approaches to create a descriptive model that may be applicable over a range of design situations. It examined case histories of design situations in order to study multiple, diverse design situations. To complement the case histories, it investigated the evolutionary and dynamic nature of the design process by studying a design situation over 14 months using multiple methods such as 
unstructured interviews, observation, document analysis, sociometric surveys, and critical incident interviews. To expand and verify the results of these studies, it incorporated aspects of the component approach by conducting semistructured interviews with design team members to investigate a "slice of time" in the design process. Thus design participants" perceptions of reality and actual behaviour as they unfold during the design process, design participants ${ }^{\circ}$ reflections on the design process and components of the design process were captured and analysed, with the goal of creating a general model that may be applicable across a range of design situations. It is a first step towards educational practices. humancomputer tools and a predictive model that facilitates knowledge exploration and collaboration during the design process.

\section{Research methods}

Four empirical, or field, studies of actual design situations in architecture. expert systems, telecommunications, and engineering design were conducted in order to develop a model that characterizes boundary spanning roles that emerge during the design process. The design situations were chosen to encompass a diversity of design domains (architecture. software, telecommunications and engineering) and diverse settings (ranging from partially autonomous settings where participants' defined their goals and task objectives to settings structured by corporate norms and practices). This diversity may lead. perhaps, to the discovery of general patterns that may be constant over a variety of design situations. In addition, the situations provided different types of data. The first two studies used retrospective case histories of design situations. Because design situations typically last from many months to several years, case histories provided the opportunity to study multiple. diverse design situations. To complement the retrospective case history data and to extend the descriptive adequacy of the results. data from two ongoing design situations were also collected.

\subsection{Architecture field study}

The design situation in the first case history focused on the construction of a single-family house over a ten-month period in 1983. Participants in this design situation included the new house owners and their family, a construction firm with four owner-employers, and an architect and his assistant. Membership within each group was stable (i.e., it did not change during the design process). The architect's office was located in Boston, MA, USA several hours away from the construction site and the users' current home near Amherst, MA, USA. Throughout the design process, a fast-track, 'design-as-you-go' approach was used. In the fast-track approach, the architect creates plans for artefact components as 
20 Kloder, T House Houghton Mitflin Company, Boston, MA (1985)

21 mumford, E Defining systern requirements to meet business neods: a case study exam. ple' Computer Joumal Vol 28 No 2 (1985) 97-104

22 Mumtord, $E$ and MacDoneld, W B XSEL's progress: the continuing joumey of an expert system Wiley, Now York (1989) 23 Leonerd-Barton, D 'The case for imtegrative innovation: an expert system at Digital' Sloan Management Reviow, Fall (1987) 7-19

24 McDormott, J 'XSEL: A computer sales person's assistant' in J E Hayes, D Michis and $Y$ Peo (eds) Machine intelligence 10 Wiley, Now York (1982) pp 325-337

25 Ainkin, $G$ 'A whote new DEC' Computer Wortd Vol 29 No $38 \mathrm{~A}$ (1986) 14-22 construction tasks proceed. Data for the case history came from the book House $^{20}$. It was selected for analysis because of its wealth of data about communication among participants during a design situation. Descriptions of design tasks, interactions among the architect, builders, and owners (including the owners' extended family), participants' perceptions of the design situation and design outcomes are provided in the 300-page book.

\subsection{Expert systems field study}

The design situation in the second case history focused on the development of an expert system, called XSEL (the eXpert SELling assistant), that was intended to assist sales staff in configuring computer systems which satisfy customer needs. The design situation was chosen because it provided an example of a participatory software design process, focused on user needs, in a corporate setting. The end-users were sales people, their managers, and office staff at Digital Equipment Corporation (DEC) offices throughout the USA; the software designers and software engineers were employees at Carnegie Mellon University and DEC. New group members joined each group during the design process which occurred over a five-year period (1981-1985). Design methods used to create the expert system included the ETHICS (Effective Technical and Human Implementation of Computer-based Systems) participatory design method ${ }^{21}$ which prescribes activities that facilitate user involvement in the design process and an iterative/prototype design approach which prescribes a succession of development and evaluation tasks until a system contains a sufficient number of features to be labelled 'completed'.

Data for this case history included the book XSEL's progress: the continuing journey of an expert system ${ }^{22}$ which provides a history of the design situation from the perspective of a group manager, a business case study of the design situation ${ }^{23}$, published and unpublished papers by designers and developers (e.g., McDermott ${ }^{24}$ ), an article on the culture of the corporation during that period ${ }^{25}$, an interview with a participant, and several documents that describe predecessor design situations. This variety of data sources provided information about events during the design process, technical and organizational tasks, characteristics of the organizational setting, interaction among participants and participants' perspectives and interpretations of these events, tasks, organizational culture and interaction.

\subsection{Telecommunications field study}

The third design situation investigated took place in the engineering organizations of a research and development (R\&D) corporation that 
employs several thousand technical employees in the USA. This design situation was chosen because it provided an example of another design domain in a large, structured corporate environment and provided the opportunity to collect data about the design process as it evolved. The superordinate goal in this design situation was to create a telecommunications network architecture and management system that would support data, voice and video communications. The design participants included approximately 14 design participants; most participants did not work full time on the project, others worked full time for intermittent time spans. They had bachelors, masters, and doctorate degrees in disciplines such as electrical engineering. operations research and business administration, and their on-the-job experience ranged from three to 37 years. The participants worked in separate office buildings, located up to 50 miles apart, and users worked in locations throughout the USA.

Multiple, co-ordinated research methods, including unstructured interviews. participant observation, document collection, sociometric surveys. and critical incident interviews, were used to gather data about communication. Using multiple methods provided flexibility for gathering data from a range of data sources, including design participants. colleagues doing related tasks in the corporation, technical papers, meeting minutes, viewgraphs amd memoranda. Data from 41 unstructured interviews, 19 participant observation periods. 125 documents. two sociometric surveys and 14 critical incident interviews were collected over a 14-month period. This approach provided a wealth of information about the experiences and perceptions of design participants and changes over time. expressed and explained in their own languages and actions.

\subsection{Engineering design field study}

The fourth design situation analysed took place in northern Europe: its goal was to create a new sensor to be used for environmental purposes. This design situation was chosen because it provided the opportunity to study the design process in another culture. The design team included 27 participants with on-the-job expertise and technical degrees in mechanical engineering, chemical engineering, materials science, electrical engineering. software engincering, production, environmental engineering and applications, and marketing. Most design participants worked for a leading international producer of precision mechanical and electronic components. Several participants worked in different divisions within this company and were located in different parts of a large office and manufacturing complex. Other participants worked for consulting firms located in two different cities, approximately one hour's flight apart. Still other participants worked in another country for different organizations. 
Because the design participants came from different disciplines, companies and countries, this design context was rich in knowledge exploration and communication behaviours.

The purpose of the fourth study was to evaluate and expand the models that emerged from my preceding studies. To achieve this, both research methods and research setting in the fourth study were dissimilar to those in the previous studies. For example, retrospective (historical) data and longitudinal data were used in the previous studies, however, in this study semistructured interviews were conducted over a three-month period with participants in a design project to investigate a particular 'slice of time' in a design project. The purpose of these interviews was to learn about participants' past experience, their current design tasks, and the nature, content and their perspective on, their current work-related interaction.

Over a three-month period, semistructured interviews were conducted with 24 participants. The average length of the interviews was two hours (their length ranged from one to eight hours), and interviews were conducted with approximately $89 \%$ of the participants in the design project (i.e., 24 out of 27 participants). Notes were taken during each interview, and I augmented these notes after each interview by drawing the interview setting, expanding on topics discussed by the interviewee, and writing my immediate impressions of the interview. Additional data came from documents such as organization charts, documentation on formal design methods used by participants, and samples of their drawings and reports that were given to me during the interviews. 154 work-related interactions or communication links were described during the interviews; 134 of which were reciprocally mentioned (i.e., participant $A$ described interacting with participant $B$ and participant $B$ described interacting with participant $\mathrm{A}$ ). This is a very high percentage of concurrence among the study participants and validates the accuracy of the research approach.

\subsection{Data analysis}

To analyse the field study data, an event sequence analysis, concept or thematic analysis and sociometric data analysis were done to discover who interacted with whom, the content of the interaction, and the meaning participants gave to the interaction. The event sequence analysis reconstructed event sequences as illustrated in Appendix 1. This reconstruction was imperative to understand each design process because participants and observers reported overlapping subsets of events, not necessarily in chronological order. The concept analysis was imperative to discover underlying concepts or themes including the meaning participants appeared to ascribe to interactions and events. Each concept derived from 
this iterative process was summarized in the form of a topic memo which cited data examples as evidence for the concept (see Appendix 2.) Sociometric data analysis identified who interacted with whom (see Appendix 3). Whole-network and individual statistics were calculated for centrality. density and other network characteristics.

Data from each study were analysed after the study was completed and interim results were reported ${ }^{26}$. Subsequent analysis built on the previous analysis in order to refine and expand research results. For additional details on data analysis, see Sonnenwald and Lievrouw ${ }^{27}$.

This multiple method approach reflects the multiperspective nature of knowledge exploration and collaboration in design perhaps more than any single method can. By looking at communication behaviour from multiple vantage points, as provided through interviews, documents, observation and surveys, we may be more likely to discover the shared experiences and perceptions of design participants. Furthermore, by analysing multiple design situations in a variety of disciplines, organizational settings and cultures. we may be more likely to discover general patterns that may be constant over a variety of design situations. As a result, new boundary spanning roles and boundary spanning roles not previously found in design situations, and the type of information exchanged via these roles during the design process was discovered.

\section{Research results}

Boundary spanning roles that emerged from the analyses of the four design situations described above are illustrated in Figure 2 (see Note 2). This illustration is a synthesis of roles found in each design situation studied. The observed boundary spanning roles are classified into five groups based on the type of boundaries they span. These are: organizational boundaries, task boundaries, discipline boundaries, personal boundaries. and roles that span multiple boundaries (see Table 2).

\section{I Organizational boundary spanning roles}

26 Sonnenwatd, D H intergroup communication in design: a pilot study' in Proceedings of the 55th American Society for information Science Meeting (1992) pp 86-92

27 Sonnenwald, $D H$ and Lievrouw, $L$ A Refloctive practice: experiences from a qualitative study of information system design' Informaatiotuktimus vol 15 No 1 (1996) 2-12 (in Finnish)
Five roles were observed with respect to spanning formal organizational boundaries: sponsor. interorganizational star. intraorganizational star. intergroup star, and intragroup star. In many design situations today. participants may come from different organizations within a company or even different companies, and may have additional job responsibilities outside the design project. In addition. membership in the design team may change over time. Therefore, organizational boundary spanning roles may have to be explicitly addressed during the design process because the 
Figure 2 Knowledge exploration roles in design and their relationships among organizational and lask structures, disciplines and individuals.

28 march, J and Simon, H Organizations Wiley, Now York (1967)

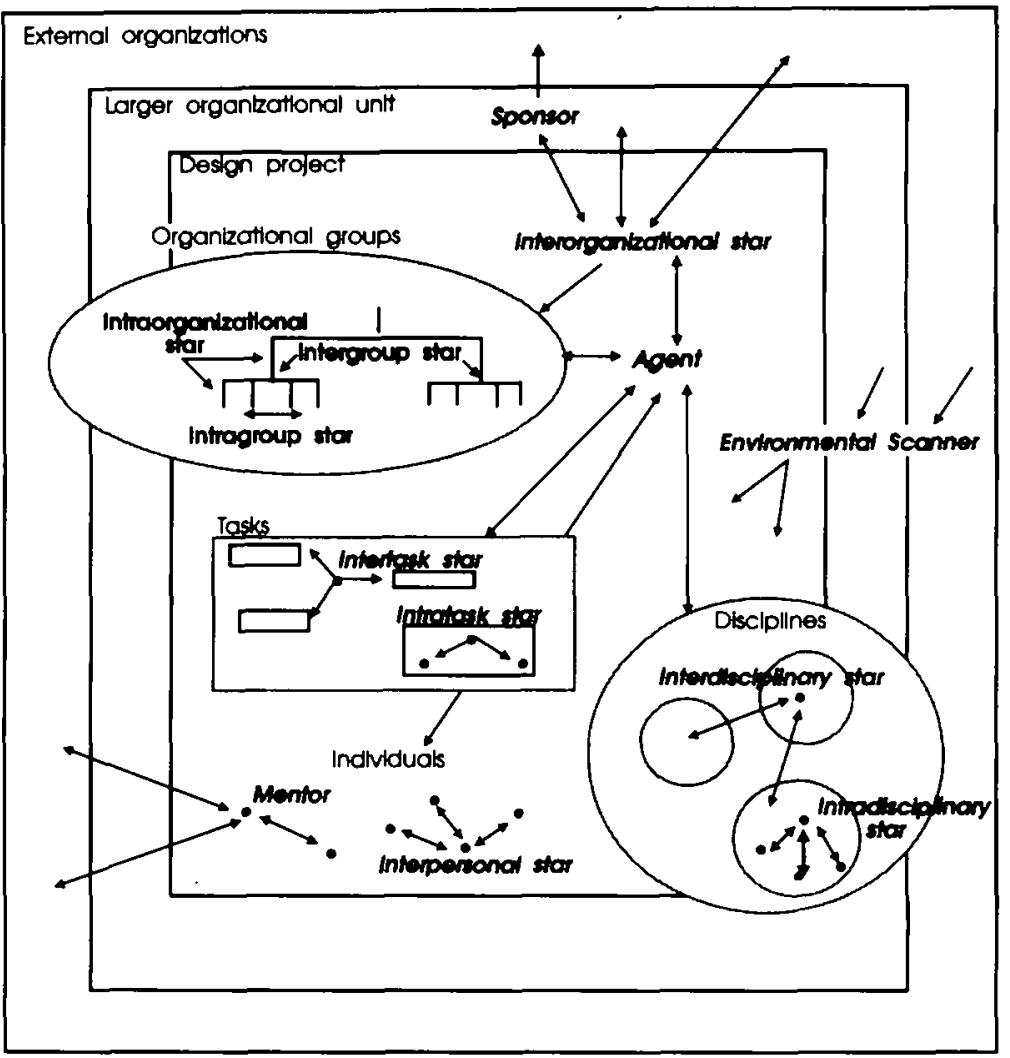

traditional organizational structure may not provide them for the design team.

The purpose of the sponsor role is to help secure acceptance and funding for the design project within the larger organizational unit, and to help ensure the design project's goals and strategies match the organization's goals and strategies. This role has been observed in other organizational contexts $^{28}$, and often the sponsor is not focused on the day-to-day issues of the design process but the role is included here for completeness.

The purpose of the interorganizational star is to interact with others in the larger organizational unit(s) and relevant external organizations in order to come to an understanding about how the design project (and possible future design projects) can meet the larger and external organizations' goals and strategies. During these interactions, the design project's goals, plans, budget, and tasks as well as the larger organizations' goals and strategies are discussed. The interorganizational star may also act as a 


\begin{tabular}{|c|c|c|}
\hline Boundary type & Role & Itscription \\
\hline \multirow[t]{5}{*}{ Organization } & Sponsor & $\begin{array}{l}\text { Helps secure acceptance and funding of the design project and } \\
\text { artefact in the larger organizational unit(s) and external unit(s) }\end{array}$ \\
\hline & $\begin{array}{l}\text { Interorganizational } \\
\text { star }\end{array}$ & $\begin{array}{l}\text { Interacts with others in the larger organizational unit(s) and } \\
\text { external unit(s) to ensure a match with the design project s goals } \\
\text { and strategies }\end{array}$ \\
\hline & Intergroup star & $\begin{array}{l}\text { Plans and co-ordinates activities across groups and represents } \\
\text { their group in planning discussions }\end{array}$ \\
\hline & $\begin{array}{l}\text { Intraorganizational } \\
\text { star }\end{array}$ & $\begin{array}{l}\text { Filecs and transmits organizational project information across } \\
\text { hicrarchical organizational levels within the design project }\end{array}$ \\
\hline & Intragroup star & Facilitates interaction among group members \\
\hline \multirow[t]{2}{*}{ Task } & Intertask star & $\begin{array}{l}\text { Facilitates interaction and negotiates conflict between people } \\
\text { doing different design tasks }\end{array}$ \\
\hline & Intratask star & Facilitates interaction, and co-ordinates activities, within a task \\
\hline \multirow[t]{2}{*}{ Discipline } & Interdisciplinary star & $\begin{array}{l}\text { Integrates knowledge from different disciplines and domains to } \\
\text { create solutions to design problems }\end{array}$ \\
\hline & Intradisciplinary star & Transmits information about new developments within a discipline \\
\hline \multirow[t]{2}{*}{ Personal } & Interpersonal star & Facilitates interaction among individuals \\
\hline & Mentor & Filters and transmits career information to individuals \\
\hline \multirow[t]{2}{*}{ Multiple } & $\begin{array}{l}\text { Environmental } \\
\text { sanner }\end{array}$ & $\begin{array}{l}\text { Iransmits information from outside the design context, but } \\
\text { relevant to the design context, to design participants }\end{array}$ \\
\hline & Agent & $\begin{array}{l}\text { Facilitates interaction and arbitrates conflict among all design } \\
\text { participants }\end{array}$ \\
\hline
\end{tabular}

filter and share knowledge about the larger organizations' goals and strategies with the organizational groups within the design project. These responsibilities are often part of the formal organizational position titled 'project leader'. Membership in a formal 'steering committee' is a strategy used by interorganizational stars to gain access to decision makers in other organizations.

Intraorganizational stars transmit and filter information about the project's goals and subgoals. plans, tasks. and detailed budget information among formal organizational levels within the design project. A person in this role might have a formal organizational title such as 'group leader'. In several design situations analysed, when participants did not receive this type of information they felt their own job performance was suboptimal and reported high job dissatisfaction. Strategies used by intraorganizational stars include frequent (e.g., daily) interaction with group members to satisfy their information needs.

Intergroup stars discuss design plans and tasks with other intergroup stars in a design situation. They represent their group in these interactions, co-ordinating activities and strategies across groups in the design team. Discussion topics during formal intergroup meetings include results. 
failures, unexpected events and alternate plans, as well as organizational contexts when intergroup stars belong to different organizations. Formal meetings may also be augmented by informal gatherings, such as dinners where interpersonal themes dominate, informal discussions about design plans, and temporary exchange of group members to foster group interaction.

Intragroup stars facilitate interaction among members of their group. The purpose of this interaction is to provide socio-emotive support to group members. Intragroup stars help resolve conflicts that may arise among group members, encourage group members to support decisions made by the group's intergroup and intraorganizational stars, and help new or disenfranchised members become full members in the group.

\subsection{Task boundary spanning roles}

Conflict may occur between design participants when their interrelated tasks have different priority measures and/or conflicting constraints. In these instances, task co-ordination and negotiations concerning interfaces between artefact components etc. are assumed by an intertask star. For example, in one design situation when a prototype subcomponent needed to be manufactured, design team members would call an intertask star (who was physically located hundreds of miles away) and ask him to ask the manufacturing personnel (who were located several hundreds of feet away from the design team members' offices) to make the part. The manufacturing personnel would make the part, ship it to the intertask star, who would then ship it the design team members who actually needed the part. Strategies used by intertask stars include asking design participants to create more than one solution and to make notes about the pros and cons of their ideas while creating solutions, keeping design constraints open for as long as possible, and sharing exciting results among participants. Other strategies include formal methods such as explicit task assignments, periodic oral and written task progress reports, and presentation and evaluation of completed tasks to design participants.

Intratask interactions includes discussion about how a task can be done or how problems that occur can be solved. As one participant explained, 'We discuss problems. He has more experience that me but often he wants to know what I've been thinking. I tell him . . . then he says, you could be right but it could also be this too.' A strategy used by intratask stars is timely sharing of task information, including results, associated data, and advantages and disadvantages of alternatives. As one intratask star explained, 'We're working so closely together, we exchange information more than once per day. We share all information with each other'. 


\subsection{Discipline boundary spanning roles}

Interdisciplinary stars interact using knowledge from their disciplines to create new knowledge and solve design problems. In describing interaction with another interdisciplinary star, a person explained. 'together we can come up with interesting concepts and solutions. . . it's intellectually inspiring ... very rewarding. Strategies used by interdisciplinary stars are often proactive in nature. For example, several interdisciplinary stars reported that they constantly monitor others facial expressions, attentiveness and questions. to determine if they are being understood. They also reported that they try to awoid using specialized language. seek to find parallel concepts in other domains to explain their perspective, and use neutral language. e.g. say I think it's not practical because . . instead of saying 'ou can't ...

Interdisciplinary sars provide information about new knowledge and developments within their discipline to others in the same discipline. Intradisciplinary stars may provide this information based on implicit or explicit information needs. For example, in one design situation. an intradisciplinary star read trade newspapers, journals and electronic bulletin boards. and provided his colleagues with regular. periodic summaries of new developments in their discipline. In another situation. colleagues in the same discipline met informally to obtain feedback on their design decisions.

\subsection{Personal boundary spanning roles}

The purpose of the interpersonal star is to facilitate interaction among individuals on the design team. Interpersonal discussions atout the weather, families, hobbies, personal experiences, world events etc. help participants to learn about each other's language and preferred interaction style. and estahlish personal bonds. This knowledge can be helpful when discussing potentially more difficult subjects such as design constraints. As one participant reported. 'Interpersonal interaction is very important in at creative climate. It makes you more open to people and ideas and not afraid of making mistakes'. To some extent every design participant may assume this role at times because interpersonal discussions are a common activity, and thus, perhaps the role should be classified as a strategy, or skill. that is part of every role. However. I list here it as a separate role because of its importance in the design process.

A memor provides individual design participants with information from the larger organizational unit(s) and external organizations that might benefit the individual's career. This type of information could be job and 
career opportunities, colleagues interested in similar problems, and services and benefits provided by the organization. Another purpose of the mentor role is to promote, or advertize, individual's achievements in other organizational unit(s). Mentors often initiate discussions concerning career issues with individuals to learn about their goals, priorities and constraints and, interpreting their needs, provide individuals with feedback and career information. This role has been identified elsewhere ${ }^{28}$, however, it may have particular importance to design due to the transitory nature of design teams; participants need to consider other job possibilities during the design process because their membership in the design project, or the design project itself, may be of limited duration.

\subsection{Roles that span multiple boundaries}

The purpose of the environmental scanner is to provide design participants with information from outside the design situation. This information can be about related competitive products, product components that could be used in the artefact, new design methods and tools, potential customers, or potential business partners or consultants. The information provided by the scanner is based on implicit and explicit needs of design participants. For example, one scanner asked friends and colleagues for information about product components (outside his area of professional expertise) which he felt might be useful to other members of the design team. I have previously referred to this role as a gatekeeper role. However, due to the proprietary nature of a design situation, e.g., when possible patents or trade secrets are under development, environmental scanners do not perform all gatekeeping functions, in particular, they do not provide information about the design context to people external to the design context.

The purpose of the agent role is to facilitate interaction among all design participants, arbitrating conflict among participants and ensuring that their information needs are met to enable them to complete their tasks. The agent in the expert design team defined his role as: 'a convener' facilitator: I will help define agenda items, keep the group focused on its task; ask critical questions; mediate conflict, and ensure that the group meets its work objectives and time targets ${ }^{29}$. Another agent defined his role as 'seeing that everyone has the information they need to do their job'. Agents may be interorganizational stars as well and have the formal job title 'project leader'. Strategies used by agents include formal project status meetings at which design participants discuss their results and meetings that introduce new participants to the design context, as well as

29 Mumford, $E$ and MacDoneld, $W$ B bid., D 87 informal strategies such as looking at and discussing participants' drawings, software code, prototypes, etc. and their design decisions face-to- 
Table 3 Lowest years of professional experlence found for each role in engineering design project

\begin{tabular}{ll}
$\begin{array}{l}\text { Lower hound } \\
\text { yeurs of } \\
\text { professional } \\
\text { experience }\end{array}$ & \\
\hline 1 & Role \\
\hline 1 & Interpersonal star \\
1 & Intradisciplinary star \\
1 & Intratask star \\
1 & Intragroup star \\
8 & Intertask star \\
8 & Intraorganizational star \\
8 & Interorganizational star \\
8 & Mentor \\
10 & Agent \\
14 & Interdisciplinary star \\
25 & Intergroup star \\
\hline
\end{tabular}

face. In many respects, this role is similar to the manager role described by Mintzberg ${ }^{301}$. however, the agent need not be the leader of the design team. For example, the agent in the architecture design situation was the father (and father-in-law) of the house owners and had no formal task or job responsibility in the project.

In conclusion, design participants may assume one or more of these roles and may change roles during the design process (see Note 3 ). However. data from the engineering design study suggest that strategies and skills needed to perform the majority of boundary spanning roles are acquired on-the-job, over the course of many years of professional experience (see Table 3). For example. in the engineering design project (see Note 4), the interpersonal, intradisciplinary. intratask, intragroup. and intertask roles were assumed by many participants whose minimal professional experience was one year. This appears to indicate that the skills needed for these roles were most likely obtained during the participants' formal education. However, the intraorganizational star, interorganizational star, mentor and agent roles were assumed by participants who had a minimum of eight years of professional experience: and the interdisciplinary star, intergroup star. and environmental scanner roles were assumed by participants who had, at the minimum, an even greater number of years of professional experience. This appears to indicate that design participants learned the strategies and skills for these roles after many years of work experience.

30 Mintzberg, $H$ Mintzberg on management Free Press. New York (1989)

\section{Discussion and future research}

Knowledge about communication roles in the design process may provide 
31 Hubke, $v$ Engineoring do sign Butterworth Scientific, London (1980)

32 Yourdon, E Managing the system life cycle Yourdon Press, Now York (1988)

33 Allon, $T \mathrm{~J}$ Managing the flow of tochnology MIT Press. Cambridge, MA (1977)

34 Ancone, $D$ G and Celdowoll, D F 'Bridging the boundary: extemal activity and pertormance in organizational toams:, Administrative Science Quarterty, Vol 37 (1992) $634-665$ insight into possible new design methods that explicitly include communication strategies, possible new computer-based tools that support communication roles during the design process and new educational needs for our students.

\subsection{Implications for prescriptive design methods}

Design methodologies have traditionally focused on design tasks and task management (e.g., Hubka ${ }^{31}$, Yourdon ${ }^{32}$ ) and communication among design team participants has not been explicitly discussed in these methodologies. Yet we know from design participants and studies ${ }^{13.15}$ that communication is often difficult for design participants and affects project outcomes. Although communication and management research has addressed research and development situations (e.g., see Allen ${ }^{33}$ ) and product management (e.g., Ancona and Caldwell ${ }^{34}$ ) and these studies have provided insights and recommendations that have been incorporated into our design practices, they do not address the increasing and, perhaps, unique complexity found in design situations. There is a need to extend our design methodologies to explicitly include communication roles and strategies.

The results presented in this paper could be a first step towards extending our design methodologies to include prescriptive guidelines for communication roles and strategies. Although the results are descriptive in nature, they can perhaps form a basis for a prescriptive framework that identifies important dimensions of, and relationships among, design tasks, communication roles, and organizational culture and project organization. For example, data from the studies suggest that the agent role can be successfully assumed by a project manager or another team member who has organizational permission and recognized technical expertise. Other data suggest that organizational cultures that define communication as the ability to educate and control others prohibit the emergence of the agent, interdisciplinary star, intergroup star and interpersonal star roles, and this appears to adversely affect project outcomes. There is a need to develop and test hypotheses about the effect of communication roles on teams to generate prescriptive guidelines that may be applicable across a range of design situations.

To address this we have developed hypotheses that explore possible relationships among design tasks, communication roles, organizational culture and project organization. These hypotheses are currently being tested in the context of ongoing design projects in telecommunications and civil engineering design situations in the USA and Europe (see Note 5). A multiple data collection approach, including sociometric surveys, 
35 Bolkin, $N \mathrm{~J}$ and Croft, B w 'Retrieval techniques' in M E Williams (ed) Annual revew of information scrence and technoto. gy Vol 22 (1987) PD 109145 36 Fox, E A, Ingworeen, $P$ and Fidel, $R$ (eds) SIGIR 95: Pro. ceedings of the 18th Annual international ACM SIGIA Confer. ence on Research and Development in Information Retrieval ACM Press. New York. NY (1995) unstructured interviews and performance surveys. is being used to collect data about the integration of design tasks, project organization, organizational culture. communication patterns and individual and team performance. Analysis methods will include social network analysis, semantic concept analysis and multivariate analysis to identify patterns of communication behaviour and their relationships to project organization, organizational culture and team and job performance: relationships not captured in other research. The goal is to develop a prescriptive framework that explicitly addresses communication among design participants across a range of design situations that can be incorporated into our design methodologies and, ultimately, design practices.

\subsection{Implications for design tools}

When boundary spanning roles do not emerge in design situations and/or when the goals of a role are not met. problematic situations develop during the design process. For example, in one design situation, the agent role did not emerge; an artefact was never created and design participants reported that they did not want to work together on future projects. In another design situation, when an intraorganizational star did not transmit project organization and project status information to his group members, they reported job dissatisfaction and suboptimal task performance. Communications and information technology have the potential to support communication roles during the design process. Of particular interest to us is information retrieval (IR) technology which has the potential capability to search and provide access to relevant information from a wide variety of diverse sources. This may help augment human boundary spanning activity and support knowledge exploration and integration during the design process.

IR technology allows users to input a sequence of words (a query) that represents their information need. A variety of algorithms ${ }^{35.3 n}$ are available to determine if documents or representations of documents in databases are relevant to the query, i.e.. they can potentially satisfy the user's information need. This is a component of many boundary spanning activities presented in this paper, e.g., intradisciplinary stars and environmental scanners searched information sources and provided colleagues with summaries and pointers to new technical developments relevant to their design tasks and interdisciplinary stars searched for information in other disciplines to help create new design solutions.

However, there are several limitations to IR technology that must be addressed in order to support these types of activitics. Today IR tools require that queries consist of terms, such as the author names, keywords 
37 Callen, J P, Croft, W B and Broglio, J 'TREC and TIPSTER experiments with INQUERY' in formation Processing and Man. agement Vol 31 No 3 (1995) 327-344

38 Cald, W R, Dumals, S T and Gallant, $S$ I 'Learned vectorspace models for document retrieval' Information Processing and Management Vol 31 No 3 (1995) $419-429$

39 Losee, $R$ M and Hans, S Sublanguage terms: dictionaries, usage and automatic classification Joumal of the American Society of information Scionce Vol 46 No 7 (1995) 519-529 40 Adzhilev, V D, Beynon, $W$ M. Cortwright, A J and Yung, $Y$ P 'A computational model for muli-agent interaction in concurrent engineoring', in Procosdings of CEEDA 94 (1994) po 227-232 and/or standard subject headings used in a discipline. This is problematic in multidisciplinary and dynamic situations because terminology differs across disciplines and task situations; the same term or query may represent different concepts and information needs across disciplines or task situations (and the same concept may be represented by different terms across disciplines and task situations). For example, consider the query: 'show me drawings of the pump'. An interorganizational star who has this query and is preparing for a meeting with end-users may need access to drawings that show the pump and its application in the end-user's domain; whereas an intertask star responsible for creating the pump's manufacturing process may need access to CAD/CAM drawings of the pump and data that specify the pump's manufacture. Furthermore IR algorithms, such as probabilistic reasoning ${ }^{37}$ and latent semantic indexing ${ }^{38}$, work well on full text or text abstracts but little is known about how they perform with the variety of resources, such as handbooks. design examples, trade magazine and newspaper advertisements and articles, project management data, internal project documents and reports, design drawings and other graphical representations, typically used by design team members.

We are currently planning research to determine how these issues may be resolved. Emerging research that examines differences in terminology across disciplines ${ }^{39}$ and new programming paradigms that facilitate creating applications based on observations of behaviour ${ }^{40}$ may provide insight towards possible solutions.

\subsection{Implications for education}

Traditional approaches to education in many design disciplines, such as information systems, teach students how to communicate with colleagues in their discipline, including the use of specialized terminology, through group projects in which two or more students work together, required reading in their discipline, and interaction with the teacher and guest speakers. As discussed above, data from the engineering design study suggest that skills for the majority of boundary spanning roles are acquired on-the-job, over the course of many years of professional experience (see Note 4). This suggests that the increasing importance and the complexity of knowledge exploration and collaboration during the design process may require education about communication roles not currently presented in design curricula.

To address this need, I have added a component on communication and boundary spanning to a master's level information systems analysis course at the University of North Carolina at Chapel Hill, USA. To introduce the 
41 Schón, D A Designing as reflective conversation with the materials of a design situation: Design Studies Vol 5 No 1 (1992) 3-14

42 Heoptors, A Co-learning in participative systems design. PhD thesis, Lund University. Sweden (1994)

43 Bucciaretll, L L 'Engineering design as negotiation - a design exercise' (unpublished manuscript) MIT, Cambridge, MA (1988) students to the importance of communication during the design process. they participatc in a group exercise that builds on Schön`s work ${ }^{+1}$ and requires groups of four to collaboratively build anything using I cegos without using verbal or written communication. In this exercise the students spend five minutes discussing how they will collaborate during this task but not what they will build; they spend the next 15-20 minutes huilding an artefact. Depriving students of verbal and written communication is a powerful way to introduce them to the importance of communication in design.

This exercise is followed by a lecture that introduces communication theory, general communication skills (as also proposed by Hagefors ${ }^{42}$ ) and boundary spanning roles and skills presented in this paper. These roles and skills are re-enforced through a simulation that provides students the opportunity to practice boundary spanning strategies. In the simulation, adapted from Bucciarellit? groups of four students must design a two-dimensional habitat for aliens on another planet. Each group member is given both unique and overlapping information about the artefact context. design process and scientific knowledge needed to complete the task. (iroups who apply boundary spanning roles in this exercise report higher satisfaction with their solution and design process. and often create more than one 'correct' solution.

Teaching students the communication roles presented in this paper and re-enforcing them through exercise is a first step in developing techniques to teach students how to participate in multidisciplinary design situations. Other possible approaches include multidisciplinary design courses and exercises which include students from a variety of (design-related) disciplines and potential end-user professions. such as business, engineering, computer science, information science and psychology. Classroom experience interacting with students from other disciplines may enhance students future job performance and the quality of design outcomes.

In conclusion, many open research questions remain with respect to knowledge exploration and collaboration in design. Challenges include determining if education courses, tools and design methods can be developed that support a range of design tasks and contexts. As a first step towards these goals, the research presented here investigated boundary spanning communication roles that appear to support knowledge exploration and collaboration during the design process.

\section{Acknowledgments}

I wish to thank the design participants for sharing their experience with 
44 Sonnenwald, D H 'Contested collaboration: A descrip. tive model of intergroup communication in information system design', Information Processing and Management Vol 31 No 6 (1995) 859-877 me; Nick Belkin, Carol Kuhlthau, Leah Lievrouw, Valerie Manusov and Scott Robertson for their generous help during the initial studies; Jacob Buur and Annelise Mark Pejtersen for facilitating the fourth study; Micheline Hancock-Beaulieu, Bob Losee, Jacob Norbjerg, Annelise Mark Pejtersen, Barbara Wildemuth and the anonymous reviewer for comments on drafts of this paper; Meurig Beynon, Elfreda Chatman, Evelyn Daniel and Steve Russ for fruitful comments; and colleagues and students at the Royal School of Librarianship in Copenhagen, Denmark, University of Tampere, Finland and Danfoss A/S who participated in, and provided feedback on the simulation exercises.

The first three field studies were supported by the Bellcore Support for Doctoral Education Program and, in part, by the American Society for Information Science and Institute of Scientific Information Dissertation Scholarship. The fourth field study and analysis was supported by the National Science Foundation Grant No. INT-9301720, the North Atlantic Treaty Organisation under a grant awarded in 1993 and Risø National Laboratory, Denmark.

\footnotetext{
Notes

1 The term, design process, is used throughout this paper to denote the time from when the superordinate goal to create an artefact, or system or product, has been established through the completion of the artefact and its manufacture or acceptance, and ideally, use by customers or end-users.

2 The term, star, is used in communication literature to denote roles that have many links, or communication paths, to other roles. To avoid inventing new terminology, the term star is used with a similar meaning here. 3 For a discussion of communication networks that appear to emerge among these rotes during the design process, see Sonnenwald"4.

4 In this design situation team members had not worked together previously and did not have first-hand knowledge about one another's skills because they came from different organizations and disciplines. Therefore role emergence was not dependent on previous relationships.

5 This study is being done in collaboration with Leah A. Lievrouw.
} 


\begin{tabular}{|c|c|c|}
\hline Timeframe & Event(s) & $\begin{array}{l}\text { Source } \\
\text { (Kidder') } \\
\text { Page no. }\end{array}$ \\
\hline Summer, autumn ' 82 & Jonathon and Judith (owners) bicycle to look at houses in the area & 14 \\
\hline January ' 82 & Bill (architect) starts own business & 22 \\
\hline Late January ' 83 & Jonathon and Judith called architect & $14,52 \mathrm{ff}$. \\
\hline \multirow[t]{3}{*}{ February 83} & Bill still working at his old firm & 59 \\
\hline & Bill visited Jonathon and Judith to discuss ideas for the housc & $52 \mathrm{ff}$. \\
\hline & Bill creates plans for hids & $31-3.3$ \\
\hline March 1. 8.3 & Jim. Ned and Richard reviewed Bill's plan. developed plan & $23-24,31$ \\
\hline March-Mid-April '83 & Meetings to discuss house plans & $32-37$ \\
\hline Mid April ' 83 & Groundbreaking & 6 \\
\hline Mon. in April ' 83 & $\begin{array}{l}\text { Jim proposed final price to Judith and Jonathon } \\
\text { (1 week after groundbreaking) }\end{array}$ & 38 \\
\hline Late April 83 & $\begin{array}{l}\text { Meeting between Jim. Judith and Jonathon to discuss contract } \\
\text { price and dates }\end{array}$ & $41-47$ \\
\hline Several days later & Construction contract signed & 46 \\
\hline Early May 83 & Concrete foundation poured & 84 \\
\hline May 17,83 & Apple Corps began building & 103 \\
\hline
\end{tabular}

Creation of time charts reconstructed event sequences. This reconstruction was imperative because participants and observers seldom reported events in chronological order and often reported overlapping subsets of events. These charts were used to create summaries of communication behaviour during events.

\section{Appendix 2 Excerpts from a topic memo}

Perceptions of the formal (corporate) design process

The stated purpose of the formal (corporate) design process is to bring people with diverse skills, such as knowledge about corporate and client strategic directions, marketing/economics, technology and user needs. together to create solution plans. Group 1 would like the process to yield dollars to build the product (e.g. . 31 October, A. p. 4 states completion of the formal process allows you to stop being subversive about how you re spending your budget.) Group 2 would like the process to allow them to create a product and receive recognition for their creation (e.g. 17 September, B). Group 3 would like the formal process to produce products with features they need (24 July, X meeting). However. I did not find any participant satisfied with the formal process. It was perceived as an 'impedance' ( 28 June. A, p. 1): 'no pass filter'. 'frustration and annoyance'. 'decisions/arguments based on process not technical or business reasons' (29 June, E, p. 7); a 'gatekeeper' and 'hurdle' (24 October, C. p. 5); and 'genuflecting' (24 September, D. p. 4). Participants reported making end runs around the formal corporate process and the formal review process. Reports of end-runs include

- 20 June interview with $E$. E had A go directly to customers to get their support outside of the formal process.

- 28 June interview with A. Put project costs under another budget category.

- 17 September. B reported that F rewrote sections of the X1 document (an output/milestone of the formal process) because they did not agree with the result written/discovered by other participants in the formal process

- 24 September interview with D. D tried to do an end-run by escalating the project to top management; top management responded by sending it back to the formal process committees.

- 4 November. $G$ sent a draft of analysis results out without completing the formal review process.

Field notes (from observation and intervicws) and documents were analysed to interpretively discover underlying concepts or themes in the data. Each concept or theme derived from this interpretative process was summarized in the form of a topic memo which cited examples of language or behaviour from the data as evidence for the concept. 


\section{Team}

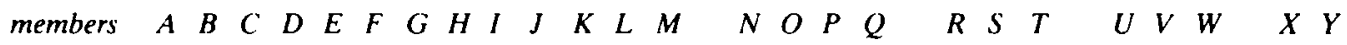

Org. group 1

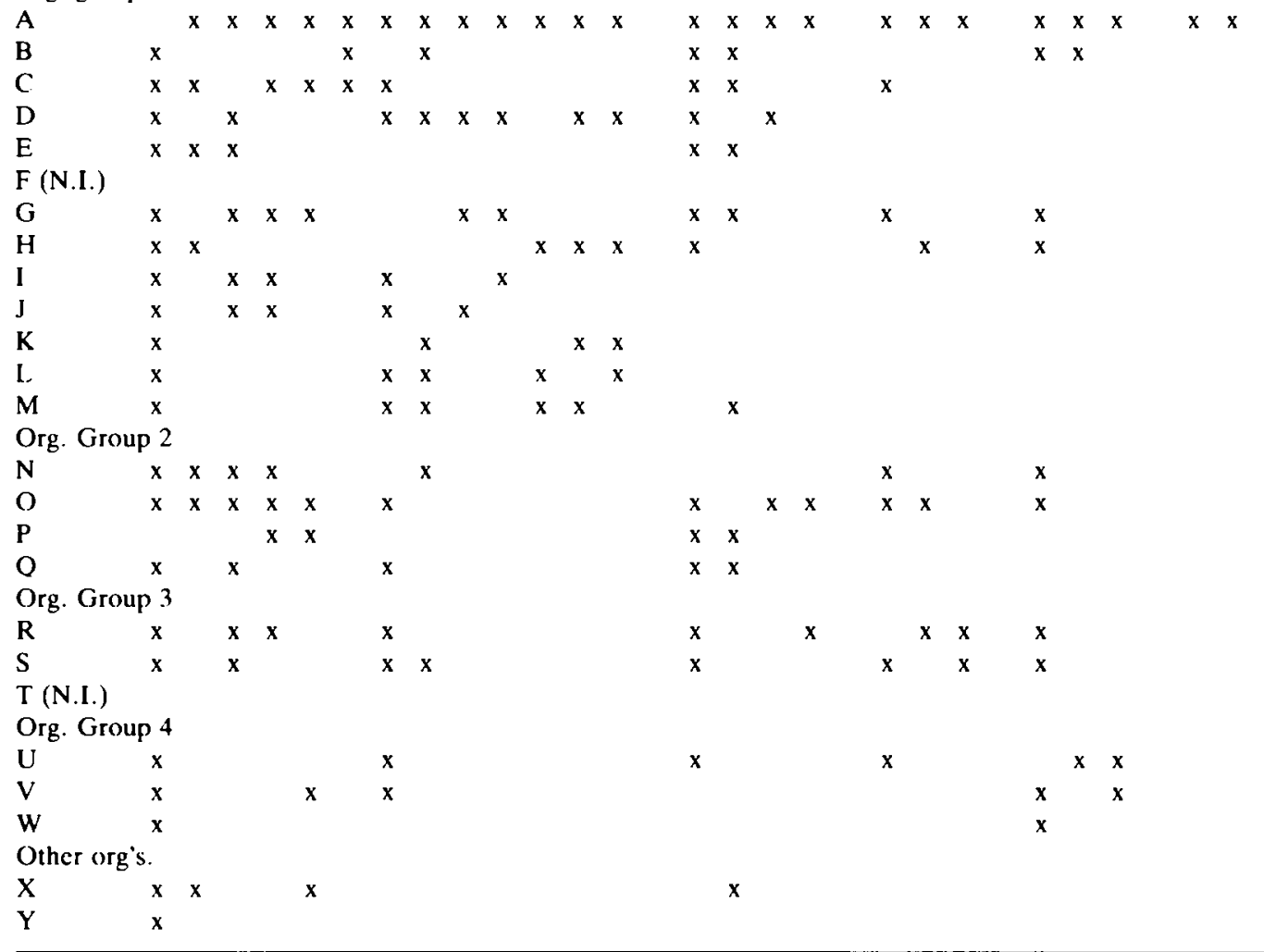

This table illustrates the interactions reported during the interviews in the fourth field study. The left-most column identifies who reported the interaction; each row identifies the people that person reported they interacted with. Study participants are grouped according to their formal organizational group. Team members not interviewed are identified with 'N.I.' next to their name. This matrix illustrates the large number of interactions reported, and the high degree of reciprocity among the interview data. When analysed in conjunction with information about team members' design tasks, patterns of communication emerged. 